\title{
Trabajo Social con familias y conflicto familia-trabajo
}

\author{
Social Work with Families and Work-Family Conflict \\ Sagrario Segado SÁnchez-CABEZudo \\ Universidad Nacional a Distancia \\ ssegado@der.uned.es \\ Amparo Osca Segovia \\ Universidad Nacional a Distancia \\ aosca@psi.uned.es \\ Antonio LóPez PeLÁEz \\ Universidad Nacional a Distancia \\ alopez@der.uned.es
}

Recibido: 26/06/2012

Revisado: 08/04/2013

Aceptado 18/04/2013

Disponible on line: 20/12/2013

\section{Resumen}

El artículo analiza el efecto directo y modulador del apoyo familiar sobre el burnout en el conflicto entre la familia y el trabajo en familias de clase media española, haciendo análisis separados en función del género para analizar mejor las diferencias. Las relaciones directas muestran sobre todo en las mujeres, que el apoyo familiar se relaciona negativamente con las dos subescalas del burnout. Respecto al papel amortiguador del apoyo familiar sobre los efectos del conflicto familia-trabajo se observan también diferencias de género: en los hombres como se esperaba, el apoyo reduce su percepción de falta de autoeficacia, sin embargo, en las mujeres cuando el conflicto es por estrés o tensión, el apoyo es positivo. Y si el conflicto es por tiempo, el apoyo es negativo, mostrando un efecto reverso. Estos resultados confirman la importancia de atender a un conflicto que se perfila como un estresor importante y cada vez más prevalente en el ámbito de los servicios de asistencia social a las familias. Un estresor que ha aumentado su presencia como consecuencia de la actual crisis económica.

Palabras clave: Conflicto trabajo-familia/familia-trabajo, conflicto por tiempo, conflicto por tensión, apoyo social familiar, burnout.

\begin{abstract}
The article analyses the direct and modulating effects of family support on burnout in the work-family conflict in Spanish middle-class families. The analysis has been separated by gender in order to better examine differences. Direct relationships in the data show that family support is negatively related to the two subscales of burnout, especially in women. Regarding the buffer effect on work-family conflict, gender differences were found: in men, as expected, support reduced the perception of lack of self-efficacy, however, in women, when the conflict was due to stress or tension, the support had positive effects. If the conflict was due to time, a reverse effect could be found and support was negative. These results confirm the importance of addressing a conflict that is emerging as a major stressor and increasingly prevalent in the field of social services to families. It is a stressor that has increased its presence as a result of the current economic crisis.
\end{abstract}

Keywords: Work-family/family-work conflict, conflict in time, conflict in tension, social family support, burnout.

Referencia normalizada: Segado Sánchez-Cabezudo, S., Osca Segovia, A., y López Peláez, A. (2013): «Trabajo Social con familias y conflicto familia-trabajo». Cuadernos de Trabajo Social, 26(2): 253-264.

Sumario: Introducción. 1. Familias y Trabajo Social. 2. Conflicto versus apoyo familiar. 3. Método. 4. Resultados. 5. Conclusiones. 6. Referencias bibliográficas. 


\section{Introducción}

En la crisis económica actual, la institución familiar se ha visto profundamente afectada. Hay varios factores que influirán de forma decisiva en el grado de vulnerabilidad de las familias, incluyendo el tipo de empleo o el desempleo de los padres, los problemas de salud, el conflicto generacional e intergeneracional, el abandono escolar, etc. De manera específica en este artículo, entre los muchos riesgos y desafíos que enfrentan las familias actualmente, constatamos que el conflicto entre familia $y$ trabajo se ha convertido en uno de los cinco riesgos principales emergentes en el mundo del trabajo (Agencia Europea para la Seguridad y Salud en el Trabajo, 2009). Este conflicto afecta a un gran número de familias y cataliza numeras situaciones de exclusión social. La literatura existente al respecto nos dice que varones y mujeres parecen afrontarlo de forma diferente.

En el estudio del conflicto entre la familia y el trabajo vamos a analizar el papel que juega, en la vulnerabilidad de las familias de clase media en España, un colectivo cuya movilidad social descendente ha aumentado, en gran parte debido al periodo de crisis que atravesamos.

\section{Familias y Trabajo Social}

Una cuestión de necesario abordaje en la disciplina del Trabajo Social es la evaluación de las familias con las que se trabaja, especialmente en estos momentos de crisis económica. Según el Informe FOESSA, 2007-2009 (Renes, 2009), el 12,8 por ciento de hogares ha pasado de una situación de inclusión a verse afectados por diferentes procesos de exclusión social. Una de las problemáticas más frecuentes que les afecta es el conflicto entre la familia $y$ el trabajo, 1 de cada 3 trabajadores en países, como España, Grecia o Letonia, manifiesta sentirse insatisfecho respecto a la conciliación de la familia y el trabajo (Encuesta de Calidad de Vida Europea, 2012). En sociedades laborales como las nuestras, el rol de trabajador/a y el rol de padre, madre, pareja, hijo, hija, etc., no siempre resulta compatible con los roles productivos, y muchos de los conflictos dentro de las familias tienen su origen precisamente en esa dificultad.

Las necesidades del grupo familiar se pueden clasificar en una serie jerárquica de etapas en orden de importancia. La clasificación de Kilpatrick (2008) además de esta jerarquía, nos ofrece directrices para el tipo de evaluación e intervención que debe llevarse a cabo en la perspectiva del Trabajo Social con familias, que pueden clasificarse en cuatro niveles: el nivel 1 de la jerarquía se refiere a las necesidades básicas de las familias, y se relaciona con los requisitos esenciales para la supervivencia y el bienestar. En el nivel 2, encontramos qué las familias son capaces de cumplir con sus funciones básicas relacionadas con la seguridad, la estabilidad y la crianza aunque tenga serios problemas para establecer los límites y mantener su autoridad.

Es en las familias de nivel 3 y 4, clasificadas como de clase media española, donde se va a centrar este artículo. En el nivel 3, la estructura familiar mínima está garantizada. Sin embargo, el conflicto surge por el tipo de relaciones familiares. El trabajador social identifica a menudo el nivel y la capacidad de supervivencia de estas familias como «apropiado» desde un punto de vista funcional. Sin embargo, se puede encontrar todo un entramado de estrategias defensivas arraigadas en la familia, donde la interacción es con frecuencia de tipo dominante, y deja muy poco espacio para la negociación. Al intervenir en el nivel 3 familiar, los trabajadores sociales anticipan una «oposición fuerte» sobre todo si la intervención se dirige a modificar patrones relacionales, profundamente arraigados e internalizados (López Peláez, 2010). En el nivel 4, las familias tienen necesidades básicas satisfechas y los límites y las estructuras son más o menos claros y satisfactorios. Las necesidades que se presentan en este nivel son de diferente orden. Implican por ejemplo, un nivel más alto de conciencia de la familia, de uno mismo, de los bienes existentes, o una mayor autonomía con respecto a los miembros de la familia.

Nuestra investigación, como hemos indicado, se centrará en un grupo de familias con un nivel educativo intermedio-alto (estudios universitarios), donde tanto el varón como la mujer tienen un empleo remunerado. Elegimos este tipo particular de familia por dos razones: en primer lugar, estas familias las se suele olvidar la literatura española (la investigación sobre familias en el ámbito del Trabajo Social se centra por lo general, en los casos 
sociales más graves de exclusión). Y en segundo lugar, porque cuando estas familias solicitan asistencia de los Servicios Sociales para resolver sus problemas, como rupturas familiares, desempleo u otros factores sociales, los proveedores de servicios encuentran a menudo problemas asociados, entre ellos, uno de alta prevalencia: el conflicto entre la familia y el trabajo.

\section{Conflicto versus apoyo familiar}

El conflicto familia-trabajo se ha estudiado en una aproximación bidireccional (Ayman y Antani, 2008) que distingue entre el conflicto del trabajo para la familia (CTF) y el conflicto de la familia para el trabajo (CFT). En este sentido, la investigación metaanalítica encuentra una mayor prevalencia del conflicto del trabajo para la familia (Kossek y Ozeki, 1999; Boyar, Maertz y Pearson, 2005; Mesmer-Magnus y Viswesvaran, 2005), que se explica fundamentalmente por dos razones: 1) porque el trabajo es el sustento económico de las familias; 2) porque las demandas familiares pueden resolverse con mayor flexibilidad y autonomía que las laborales. Los meta-análisis apuntan a que la influencia del conflicto entre la vida laboral y la familiar está modulada por el género y el peso de los roles sociales (Korabik, McElwain y Chappell, 2008). Una de las explicaciones más acreditadas en el tiempo que apoyan esta influencia es la hipótesis de los límites permeables asimétricos (Pleck, 1977). Plantea que las relaciones no son similares en ambos sexos y que el conflicto del trabajo para la familia es mayor en el caso de los varones, porque hay una mayor permisividad a que el trabajo interfiera en su vida familiar. Por el contrario, las mujeres experimentan más el conflicto de la familia con el trabajo, pues las creencias respecto a sus roles son más fuertes, sobre todo si tienen hijos (Parasuraman, Greenhaus y Granrose, 1992). En línea con estos resultados en España, autores como Cantera, Cubells, Martínez, Blanch y Josep (2009) encuentran que las mujeres valoran más la institución familiar, lo que coincidiría con su rol social.

En cuanto al apoyo social, se considera un elemento clave para entender la relación entre el estrés y la salud. El apoyo social para el desempeño laboral puede venir de la familia y del propio ámbito laboral, y su influencia se manifiesta el modelo de respuestas de los empleados a los estresores, y dentro de estos últimos, al conflicto entre las demandas laborales y familiares (Baruch-Feldman, Brondolo, Ben-Dayan y Schwartz, 2002). Aun así, los metaanálisis encuentran relaciones débiles entre el apoyo social y el estrés (Viswesvaran, Sánchez, Fisher, 1999) y consideran que puede haber variables moduladoras que estén influyendo y reduzcan su efecto. En la misma dirección, los estudios sobre conflicto entre familia, trabajo y apoyo también señalan la influencia de terceras variables. Por ejemplo, Matthews, Bulger y Barnes-Farrell (2010) encuentran que el apoyo familiar se relaciona negativamente con el conflicto de la familia con el trabajo, aunque sobre todo en personas de mediana edad, probablemente debido a las demandas de cuidado de los hijos. Para Greenhaus y Beutell (1985) el apoyo mejora la salud, bien reduciendo directamente el conflicto, o bien amortiguando su efecto. En cncreto, respecto al apoyo para un desempeño laboral saludable, el metaanálisis de Halbesleben (2006) muestra vínculos directos y significativos con las tres dimensiones del burnout. En cuanto al efecto modulador de la ayuda, Witt y Carlson (2006) encuentran que el apoyo organizacional percibido modula los efectos de la interferencia de la familia sobre el trabajo, de forma que si el apoyo es alto apenas influye, pero cuando es bajo tienen lo desempeñan peor quienes presentan más interferencias. Sin embargo, no hemos encontrado trabajos sobre cómo influye el apoyo familiar en las situaciones de elevado conflicto entre familia y trabajo; ni que analicen las diferencias de género, como se ha recomendado (Halbesleben, 2006). Con las evidencias planteadas las hipótesis de este estudio son las siguientes:

Hipótesis 1: El apoyo de la familia se relacionará directa y negativamente con las tres dimensiones del burnout.

Hipótesis 2: El apoyo de la familia ejercerá un papel modulador entre el conflicto entre familia y trabajo y el burnout, de forma que amortiguará sus efectos negativos, pero sólo cuando se den elevados niveles de conflicto.

Además se han realizado análisis separados para abordar las posibles diferencias debidas al género. 


\section{Método}

\subsection{Participantes y procedimiento}

La muestra es incidental y se recogieron datos en todo el Estado español. Para ello se utilizó un cuestionario y actuaron como encuestadores los alumnos de doctorado de un curso sobre esta temática. A los participantes se les garantizó la confidencialidad de los datos aportados. La muestra está compuesta por 427 personas que son miembros de «familias de dos carreras», el 51,2 por ciento mujeres. La media de edad es de 38.3 años (DT $=9.56)$ y oscila entre los 21 y los 64 anos. Casi dos tercios de la muestra tienen estudios universitarios. No hay diferencias significativas entre varones y mujeres en número de hijos (Chi Cuadrado $=.62$; $p<.96$ ), ni nivel de estudios (Chi Cuadrado $=6.70 ; p<.24)$. En cuanto a los ingresos brutos anuales por pareja, el 16,7 por ciento gana menos de 20.000 euros, el 51,6 recibe ingresos entre 20.000 euros y 40.000 euros y el 31,7 por ciento tiene ingresos superiores a 40.000 euros anuales ${ }^{1}$.

\subsection{Medidas}

El formato de respuesta de todas las variables evaluadas es una escala Likert de 7 puntos. Para evaluar el conflicto se han utilizado el cuestionario Conflicto trabajo-familia de Carlson, Kacmar y Williams (2000), concretamente las cuatro escalas que combinan las dos direcciones del conflicto del trabajo con la familia y de la familia con el trabajo, y las dos dimensiones del conflicto: por tiempo y por tensión. Para estudiar el apoyo familiar se utilizó una reducción de la adaptación al castellano de Osca y Martínez-Pérez (2002) del inventario de Apoyo familiar para trabajadores (King, Mattimore, King y Adams (1995) compuesta por 8 ítems. Por último, se ha utilizado el inventario de burnout de Maslach, Jackson, y Leiter (1996), adaptado al castellano por GilMonte (2002) y sus tres subescalas: agotamiento emocional (por ejemplo, ítem «Debido a mi trabajo me siento emocionalmente agotado»), cinismo (por ejemplo, ítem «Dudo de la importancia de mi trabajo») y falta de autoefi- cacia personal (por ejemplo, ítem «Pienso que no estoy haciendo una contribución significativa a los objetivos de mi empresa»). Las puntuaciones medias, las fiabilidades y las correlaciones entre variables para varones y mujeres se representan en la Tabla 1.

\subsection{Resultados}

Para someter a prueba las hipótesis del estudio y comprobar las relaciones entre conflicto, apoyo familiar y burnout, se han llevado a cabo ecuaciones de regresión jerárquica, según se recomienda, para determinar el efecto de interacción (Zedeck, 1971; Cohen, Cohen, West y Aiken, 1983). Se han realizado tres ecuaciones de regresión, una para cada dimensión del burnout. Las variables independientes se han incluido mediante el procedimiento de Introducción, en tres bloques: en el primero se introducen los cuatro tipos de conflicto, en el segundo el apoyo familiar (hipótesis 1) y, en el tercero, las interacciones entre los cuatro tipos de conflicto y el apoyo (hipótesis 2). Las ecuaciones de regresión se han hecho para varones y mujeres, lo que permite analizar las diferencias entre ambos.

Como se puede observar en la Tabla 2, en la primera ecuación y tomando como variable dependiente el agotamiento emocional en los varones, se ve que el primer bloque con las subescalas del conflicto ofrece valores significativos $(F=11.71 ; p<.001)$ y explica el 17,3 por ciento de su varianza, aunque sólo alcanzan la significación los relativos a la tensión, en las dos direcciones: trabajo-familia $(\beta=.22 ; p<.01)$ y familia-trabajo $(\beta=.32$; $p<.001)$. En el segundo bloque el apoyo familiar no es significativo $(\beta=.06 ; p>.05)$, y en el tercero, ninguna interacción alcanza la significación mínima. Los resultados, para la predicción del agotamiento emocional en las mujeres, muestran que el primer bloque explica el 26,9 por ciento de la varianza $(F=20.13$; $p<.001)$ y resultan significativos el conflicto trabajo-familia por tiempo $(\beta=.17 ; p<.05)$, el conflicto trabajo-familia por tensión $(\beta=.38 ; p<.001)$ y el conflicto familia-trabajo por tensión $(\beta=.14 ; p<.05)$. El segundo

${ }^{1}$ Dado que los ingresos de familias de clase media en España oscilan entre 18,000 euros (media baja) y 60,000 euros (medio superior). Podemos afirmar que la muestra se compone principalmente de familias clase media. 


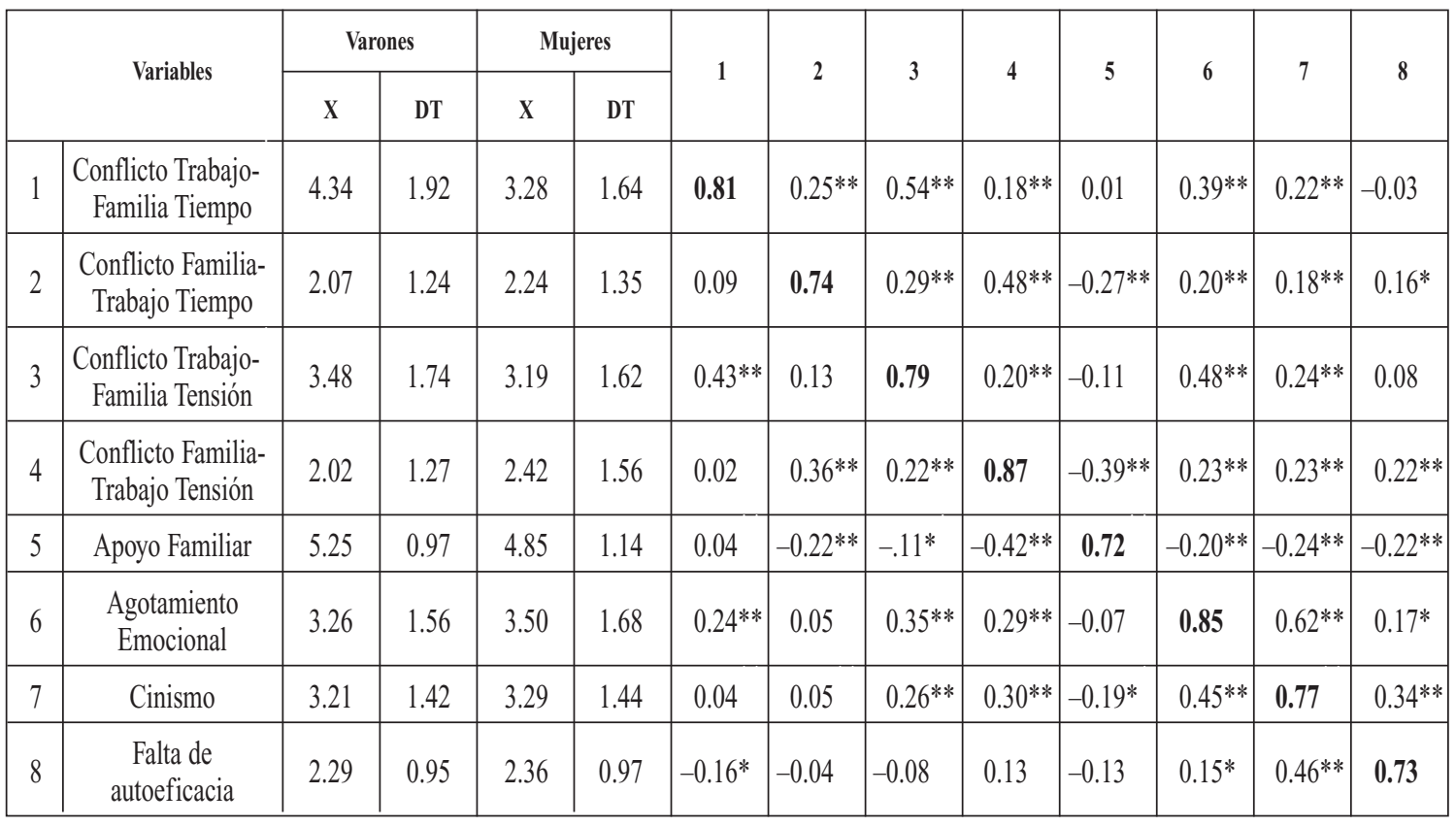

Tabla 1. Descriptivos, fiabilidades, correlaciones entre variables y fiabilidad.

$* p<0.05$; ** $p<0.01$. Escala de respuesta: 1-7. La fiabilidad de las escalas en la diagonal. Datos de los varones por debajo de la diagonal, y de las mujeres por encima de la diagonal.

\begin{tabular}{|c|c|c|c|}
\hline & $\beta$ & $F$ & $\Delta \mathbf{R}^{2}$ \\
\hline \multicolumn{4}{|l|}{ VARONES } \\
\hline 1. ${ }^{\circ}$ Conflicto Trabajo-Familia Tiempo (TFT) & 0.11 & $11.71 * * *$ & $.19 * * *$ \\
\hline Conflicto Familia-Trabajo Tiempo (FTT) & -0.12 & & \\
\hline Conflicto Trabajo-Familia Tensión (TFS) & $0.22 * *$ & & \\
\hline Conflicto Familia-Trabajo Tensión (FTS) & $0.32 * * *$ & & \\
\hline 2. ${ }^{\circ}$ Apoyo familiar (AF) & 0.06 & $9.42 * * *$ & 0.00 \\
\hline 3. ${ }^{\circ} \mathrm{AF}^{*} \mathrm{TFT}$ & 0.06 & $0.54 * * *$ & 0.01 \\
\hline $\mathrm{AF} * \mathrm{FTT}$ & -0.10 & & \\
\hline AF*TFS & 0.03 & & \\
\hline AF*FTS & 0.05 & & \\
\hline \multicolumn{4}{|l|}{ MUJERES } \\
\hline 1. ${ }^{\circ}$ Conflicto Trabajo-Familia Tiempo (TFT) & $0.17 *$ & $20.21 * * *$ & $0.27 * * *$ \\
\hline Conflicto Familia-Trabajo Tiempo (FTT) & -0.01 & & \\
\hline Conflicto Trabajo-Familia Tensión (TFS) & $0.38 * * *$ & & \\
\hline Conflicto Familia-Trabajo Tensión (FTS) & $0.15^{*}$ & & \\
\hline 2. ${ }^{\circ}$ Apoyo familiar (AF) & $-0.19^{*}$ & $17.21 * * *$ & 0.01 \\
\hline 3. ${ }^{\circ} \mathrm{AF}^{*} \mathrm{TFT}$ & 0.03 & $9.55 * * *$ & 0.00 \\
\hline $\mathrm{AF}^{*} \mathrm{FTT}$ & -0.01 & & \\
\hline AF*TFS & 0.03 & & \\
\hline AF*FTS & -0.03 & & \\
\hline
\end{tabular}

Tabla 2. Resultados de las ecuaciones de regresión para predecir el agotamiento emocional en varones y mujeres.

$* p<0.05 ; * * p<0.01 ; * * *<<0.001$. 


\begin{tabular}{|c|c|c|c|}
\hline & $\beta$ & $F$ & $\Delta \mathbf{R}^{2}$ \\
\hline \multicolumn{4}{|l|}{ VARONES } \\
\hline 1. ${ }^{\circ}$ Conflicto Trabajo-Familia Tiempo (TFT) & -0.04 & \multirow[t]{4}{*}{$7.98 * * *$} & \multirow[t]{4}{*}{$0.14 * * *$} \\
\hline Conflicto Familia-Trabajo Tiempo (FTT) & -0.09 & & \\
\hline Conflicto Trabajo-Familia Tensión (TFS) & $0.19 * *$ & & \\
\hline Conflicto Familia-Trabajo Tensión (FTS) & $0.31 * * *$ & & \\
\hline 2. ${ }^{\circ}$ Apoyo familiar (AF) & -0.12 & $6.63 * * *$ & 0.00 \\
\hline $3 .^{\circ} \mathrm{AF} * \mathrm{TFT}$ & -0.02 & $4.02 * * *$ & 0.01 \\
\hline AF*FTT & -0.02 & & \\
\hline AF*TFS & 0.10 & & \\
\hline AF*FTS & 0.01 & & \\
\hline \multicolumn{4}{|l|}{ MUJERES } \\
\hline 1. ${ }^{\circ}$ Conflicto Trabajo-Familia Tiempo (TFT) & 0.10 & \multirow[t]{4}{*}{$6.13 * * *$} & \multirow[t]{4}{*}{$0.11 * * *$} \\
\hline Conflicto Familia-Trabajo Tiempo (FTT) & 0.03 & & \\
\hline Conflicto Trabajo-Familia Tensión (TFS) & 0.12 & & \\
\hline Conflicto Familia-Trabajo Tensión (FTS) & $0.16^{*}$ & & \\
\hline 2. ${ }^{\circ}$ Apoyo familiar (AF) & $-0.23^{*}$ & $6.36 * * *$ & 0.02 \\
\hline 3. ${ }^{\circ} \mathrm{AF} * \mathrm{TFT}$ & -0.07 & $3.91 * * *$ & 0.01 \\
\hline $\mathrm{AF} * \mathrm{FTT}$ & -0.02 & & \\
\hline AF*TFS & 0.07 & & \\
\hline $\mathrm{AF} * \mathrm{FTS}$ & -0.08 & & \\
\hline
\end{tabular}

Tabla 3. Resultados de las ecuaciones de regresión para predecir el cinismo en varones y mujeres. $* p<0.05 ; * * p<0.01$; *** $p<0.001$.

bloque con el apoyo $(\beta=-.18 ; p<.05)$ también es significativo $(F=17.21, p<.001)$, pero no lo son las interacciones del tercer bloque.

En la Tabla 3 se presentan los resultados de la predicción de las puntuaciones de cinismo. Como se puede apreciar para los varones, el primer bloque resulta significativo $(F=7.98$; $p<.001$ ), y explica el 14.2 por ciento de la varianza. En este caso sólo resultan significativos los conflictos basados en la tensión en las dos direcciones: trabajo-familia $(\beta=.19 ; p<.01)$ y familia-trabajo $(\beta=.31 ; p<.001)$. El segundo bloque alcanza la significación $(\beta=.15$; $p<.05)$, aunque no llega a aumentar el porcentaje de varianza explicada respecto al paso anterior y, lo mismo sucede con el tercer bloque. Para las mujeres, los resultados muestran que el primer bloque resulta significativo $(F=6.13 ; p<.001)$ y explica el 10,9 por ciento de la varianza del cinismo, pero sólo contribuye el conflicto familia-trabajo por tensión $(\beta=.16 ; p<.05)$. En el segundo bloque, el apoyo familiar también alcanza la significación $(\beta=-.23 ; p<.05)$, pero en el tercer blo- que ninguna de las interacciones alcanza valores significativos.

La Tabla 4 representa los resultados de la predicción de las puntuaciones de falta de autoeficacia personal. Como se puede observar para los varones, el primer bloque es significativo $(F=2.57 ; p<.05)$, aunque sólo contribuye a la significación el conflicto familia-trabajo por tensión $(\beta=.12 ; p<.05)$. El segundo bloque también es significativo $(F=2.34$; $p<.05)$, pero el apoyo no aumenta la varianza explicada. En el tercer bloque, aparece una interacción significativa, entre el conflicto familia-trabajo por tiempo y el apoyo familiar $(\beta=-.134 ; p<.05)$. En la Figura 1 se comprueba que los niveles más elevados de falta de eficacia se dan en situaciones de escaso apoyo familiar, sin embargo y como se esperaba, aquellos sujetos que se sienten más apoyados por su familia se perciben más eficaces, pero sobre todo si tienen un alto conflicto familiatrabajo por tiempo.

En las mujeres del primer bloque sólo resulta significativo el conflicto familia-trabajo 


\begin{tabular}{|c|c|c|c|}
\hline & $\beta$ & $\boldsymbol{F}$ & $\Delta \mathbf{R}^{2}$ \\
\hline VARONES & & & \\
\hline 1. $\begin{array}{r}\text { Conflicto Trabajo-Familia Tiempo (TFT) } \\
\text { Conflicto Familia-Trabajo Tiempo (FTT) } \\
\text { Conflicto Trabajo-Familia Tensión (TFS) } \\
\text { Conflicto Familia-Trabajo Tensión (FTS) }\end{array}$ & $\begin{array}{l}-.07 \\
-.06 \\
-.03 \\
.13 *\end{array}$ & $2.57 *$ & $.03 *$ \\
\hline 2. ${ }^{\circ}$ Apoyo familiar (AF) & -.09 & $2.34 * * *$ & .00 \\
\hline $\begin{array}{l}\text { 3. }{ }^{\circ} \mathrm{AF}^{*} \mathrm{TFT} \\
\mathrm{AF}^{*} \mathrm{FTT} \\
\mathrm{AF}{ }^{*} \mathrm{TFS} \\
\mathrm{AF}{ }^{*} \mathrm{FTS}\end{array}$ & $\begin{array}{l}.08 \\
-.13 * \\
.02 \\
-.01\end{array}$ & $2.33 * * *$ & $.04 *$ \\
\hline MUJERES & & & \\
\hline $\begin{array}{r}\text { 1. } \\
\text { Conflicto Trabajo-Familia Tiempo (TFT) } \\
\text { Conflicto Familia-Trabajo Tiempo (FTT) } \\
\text { Conflicto Trabajo-Familia Tensión (TFS) } \\
\text { Conflicto Familia-Trabajo Tensión (FTS) }\end{array}$ & $\begin{array}{l}-.08 \\
.05 \\
.06 \\
.12^{* *}\end{array}$ & $3.75^{* *}$ & $.05 *$ \\
\hline 2. ${ }^{\circ}$ Apoyo familiar (AF) & $-.11 *$ & $3.28 * *$ & .01 \\
\hline $\begin{array}{l}\text { 3. }{ }^{\circ} \mathrm{AF}^{*} \mathrm{TFT} \\
\mathrm{AF}{ }^{*} \mathrm{FTT} \\
\mathrm{AF} \text { TFS } \\
\mathrm{AF} * \mathrm{FTS}\end{array}$ & $\begin{array}{l}.14^{* *} \\
.02 \\
-.11^{* *} \\
.04\end{array}$ & $3.68 * * *$ & $.05 * *$ \\
\hline
\end{tabular}

Tabla 4. Resultados de las ecuaciones de regresión para predecir la falta de eficacia en varones y mujeres. $* p<.05 ; * * p<.01 ; * * * p<.001$.

\section{HOMBRES}

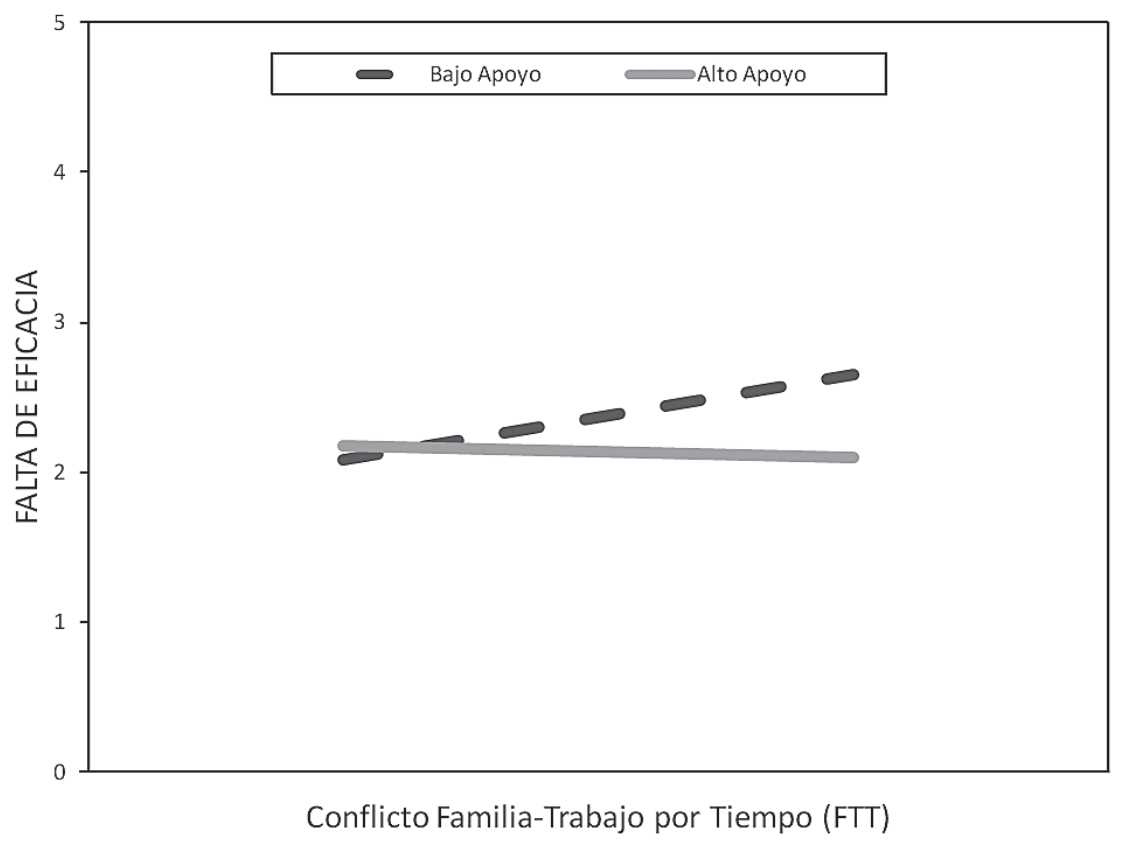

Figura 1. Interacción del apoyo familiar por el conflicto familia-trabajo por tiempo en el grupo de varones 


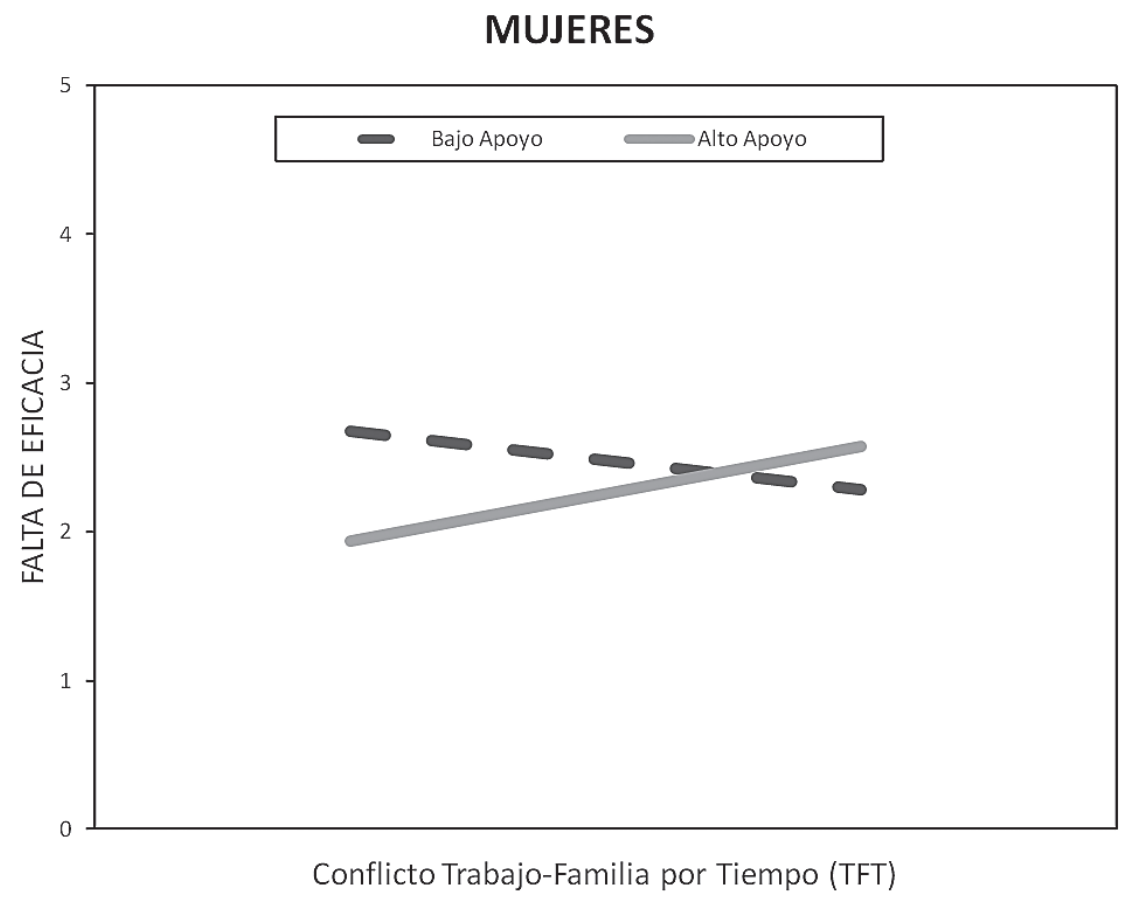

Figura 2. Interacción del apoyo familiar por el conflicto trabajo-familia por tensión en el grupo de mujeres

por tensión $(\beta=.12 ; p<.01)$; en el segundo, el apoyo familiar también es significativo $(\beta=-.11 ; p<.05)$ y aparecen dos interacciones del tercer bloque $(F=3.68, p<.001)$ : el conflicto trabajo-familia por tensión por el apoyo familiar $(\beta=-.11 ; p<.01)$ y el conflicto trabajo-familia por tiempo por el apoyo $f a-$ miliar $(\beta=.14 ; p<.01)$.

La Figura 2 muestra que las mujeres presentan menos eficacia con elevado conflicto familia-trabajo por tensión y escaso apoyo, sin embargo y como se esperaba en situación de elevado conflicto, el apoyo familiar actúa reduciendo de forma significativa su percepción de falta de eficacia personal.

Sin embargo, en la Figura 3 se observa que, cuando el conflicto es bajo, el apoyo familiar apenas influye en sentimiento de falta de eficacia; pero cuando el conflicto es elevado, aquellas mujeres que se sienten más apoyadas son las que se perciben menos eficaces, con lo que el apoyo ejercería un efecto reverso.

\section{Resultados}

El objetivo de este trabajo es analizar el papel de apoyo familiar en el burnout y, concreta- mente se han puesto a prueba dos hipótesis: la influencia directa y negativa del apoyo familiar sobre el burnout y la interacción del apoyo familiar con el conflicto familia-trabajo. La interacción significaba que un elevado apoyo amortiguaría los efectos negativos de elevados niveles de conflicto. Además, y dado que desde diferentes perspectivas teóricas, la investigación recomienda considerar el género en las relaciones analizadas, por ello se han efectuado análisis separados para varones y mujeres (o viceversa) buscando identificar patrones posibles de respuesta.

Los análisis confirman la primera hipótesis, que predecía una relación directa y negativa entre el apoyo familiar y las tres dimensiones del burnout, y se observan diferencias en función del género. En línea con la bibliografía revisada, son las mujeres las que más se benefician del apoyo familiar probablemente, como se ha señalado, por coincidir con su rol social (Westman y Etzioni, 2002). Los resultados muestran que en las mujeres el apoyo familiar se relaciona negativamente con las tres dimensiones del burnout, mientras que en los varones sólo se aprecia una correlación negativa 


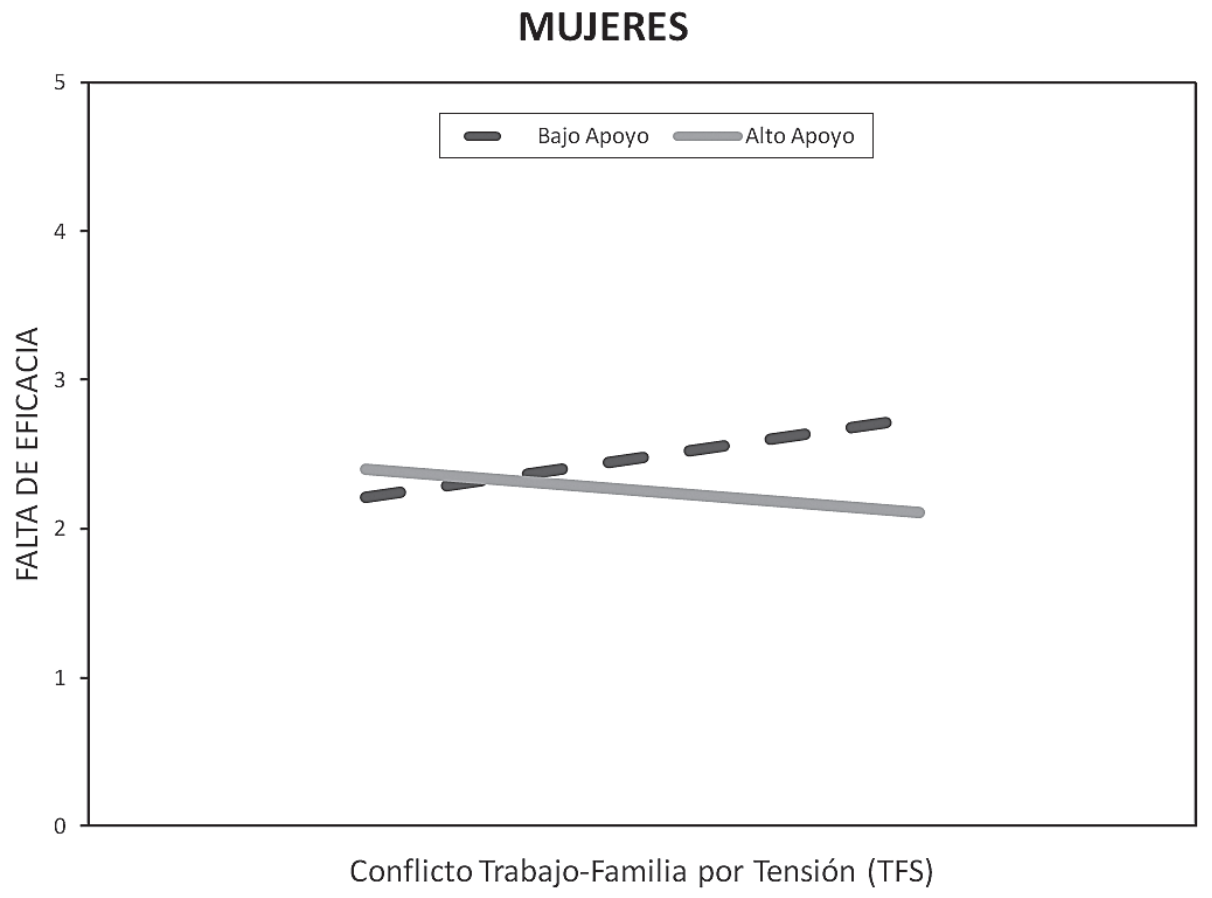

Figura 3. Interacción del apoyo familiar por el conflicto trabajo-familia por tiempo en el grupo de mujeres

con el cinismo, y de menor magnitud. No obstante, cabe destacar que los varones responden que se sienten mucho más apoyados por su familia que ellas.

La hipótesis segunda — el apoyo amortigua los elevados niveles de conflicto familia-trabajo- se ha corroborado en tres ocasiones, en todas ellas explica la reducción de la percepción de autoeficacia personal. En los varones, como era de esperar, cuando el conflicto se debe a la interferencia de la familia en el trabajo y a la falta de tiempo, el apoyo familiar reduce su percepción de autoeficacia personal. La segunda y la tercera interacción significativas se observan en el grupo de mujeres y en ambos casos para explicar el conflicto en la dirección del trabajo a la familia. A ellas el apoyo familiar les ayuda o no, dependiendo de que el conflicto se deba a una cuestión de tiempo o de tensión. En el primer caso, es decir si es por falta de tiempo, el apoyo familiar no mejora su situación, sino que tiende a aumentar su percepción de falta de autoeficacia, dándose por lo tanto un efecto reverso como se encuentra con otros tipos de estresores y de apoyo (Kahn y Byosiere, 1992). Sin embargo, en el caso de que el conflicto se deba a la falta de tiempo, el apoyo familiar como se planteó, ha ejercido un efecto positivo.

No obstante, convendría que hacer dos matizaciones respecto a las interacciones. Como se ha señalado para identificar interacciones significativas es importante que las puntuaciones en las variables estén por encima de los valores medios (Cohen et al., 1983). Esto explicaría que sólo hayan resultado significativas las interacciones relativas a las dimensiones del conflicto que van en la dirección del trabajo a la familia, que son las que han mostrado puntuaciones más elevadas. Por otra parte, que las interacciones no se hayan encontrado en las dimensiones centrales del burnout, agotamiento emocional y cinismo, pero sí en la percepción de falta de autoeficacia podría deberse a que, como se ha señalado (Schaufeli y Bakker, 2004), no es una dimensión más del mismo. Según esto, nuestros resultados mostrarían que el apoyo familiar actuaría directamente sobre las dimensiones centrales del burnout agotamiento emocional y cinismo, sobre todo en las mujeres, mientras que funcionaría como modulador en la percepción de la reducción de la 
autoeficacia personal.

Otro de resultado destacable es que, en todos los análisis realizados, las variables predictoras permiten explicar mucha más varianza en el grupo de mujeres que en el de varones. Frente a los planteamientos que hablan de la segmentación entre los ámbitos familiar y laboral, en nuestros análisis constatamos relaciones pues el apoyo familiar repercute positivamente en el burnout, aunque más a las mujeres que a los varones. No podemos constatar esta información con trabajos, ya que la mayoría no distingue en función del género, como indica el meta-análisis de Amstad, Meier, Fasel, Elfering y Semmer (2011). En este sentido creemos que nuestros resultados abren una vía que conviene seguir explorando.

\section{Conclusiones}

Los resultados muestran que el conflicto entre la familia y el trabajo es cada vez más frecuente entre las familias españolas. El perfil de las familias excluidas en España ha cambiado sustancialmente si miramos los últimos datos disponibles en el período 2007-2009 (Renes 2009). Aunque las intervenciones de los Servicios Sociales en los años anteriores a 2007 se han centrado fundamentalmente en el nivel $1 \mathrm{y}$ 2 de familias, las más recientes encuentran familias con un perfil diferente como resultado de la crisis. En concreto, nos referimos a las familias donde la estructura básica familiar está garantizada, pero cuyo funcionamiento relacional dista de ser «el adecuado». Y precisamente, porque estas familias están acostumbradas a una vida estructurada, el ataque también estructural que sufren, derivado del conflicto familia-trabajo tiene un impacto aún más negativo sobre ellos. Por otra parte, debido a que están acostumbrados a proporcionar a sus hijos la atención suficiente, la imposibilidad de continuar prestando dicha asistencia cuando acceden a la zona de exclusión, consigue fracturar aún más la estructura familiar.

Como hemos visto, el estrés causado por el conflicto trabajo-familia se asocia con el agotamiento, tanto en varones como en mujeres. Sin embargo, una vez más, este conflicto se percibe más como una cuestión relativa a las mujeres y no tanto a los varones (Stier, LewinEpstein, y Braun, 2012). A la luz de estos resultados, es importante destacar dos temas que son relevantes para este estudio. El primero se refiere a la metodología, específicamente la distinción de género en las relaciones entre este conflicto, el apoyo familiar, el carácter bidireccional de dicho conflicto (el conflicto de del trabajo a la familia y el conflicto de la familia al trabajo) y sus dimensiones (tiempo y el estrés). Se han llevado a cabo muy pocos estudios de este tipo, creemos que el presente estudio, ha puesto de manifiesto una serie de relaciones interesantes en este sentido. La segunda cuestión se refiere a la posibilidad de generalizar estos datos en los procesos de evaluación en el campo del trabajo social familiar, en particular en la evaluación de las familias clasificadas como «normales» por los servicios sociales, y que sin embargo representan el mayor porcentaje de servicios/intervenciones realizadas (SIUSS, 2009). Los resultados son concluyentes y significativos: el estrés en el ámbito familiar afecta al dominio de trabajo, y esto ocurre tanto en varones como en mujeres. Pocos estudios han señalado la necesidad de programas en Servicios Sociales dirigidos a la conciliación de trabajo y familia y, para reiterarlo, los datos de este estudio apoyan esta convocatoria. En este contexto, tanto en la literatura revisada como en los resultados de este estudio se ha demostrado que el apoyo de la familia actúa como un amortiguador para el desempeño de roles en el trabajo y en la familia que resultan mutuamente excluyentes. El apoyo familiar (es decir, el apoyo de los socios, los abuelos y la familia extensa, que ayuda en el cuidado de los niños, las redes de amigos, etc.) mitiga el cansancio emocional, el cinismo y los sentimientos de ineficacia. En particular, este apoyo, reduce el estrés en las mujeres y alivia la falta de tiempo de los varones. Desde la perspectiva del trabajo social familiar, la consecuencia es clara: es necesario fortalecer el apoyo familiar mediante el diseño de estrategias para hacer que ese apoyo resulte eficaz a todos los niveles. 


\section{Referencias bibliográficas}

Adam, S., Gyorffy, Z. y Susanszky, E. (2008). Physician burnout in Hungary A potential role for work-family conflict. Journal of Health Psychology, 13(7), 847-856.

Agencia Europea para la Seguridad y la Salud en el Trabajo (2009). En http://osha.europa.eu/ en/publications/annual_report/2009full.

Amstad, F., Meier, L., Fasel, U., Elfering, A. y Semmer, N. (2011). A Meta-Analysis of Work- Family Conflict and Various Outcomes with a Special Emphasis on Cross-Domain versus Matching-Domain Relations. Journal of Occupational Health Psychology, 16(2), 151-169.

Ayman, R. y Antani, A. (2008). Social Support and Work-Family Conflict. En K. Korabik, D. Lero y D. Whitehead (eds). Handbook of Work-Family Integration: Research, Theory and Best Practices (pp. 287-304). Amsterdam: Elsevier.

Baruch-Feldman, C., Brondolo, E., Ben-Dayan, D. y Schwartz, J. (2002). Sources of social support and burnout, job satisfaction and productivity. Journal of Occupational Health Psychology, 7(1), 84-93.

Beehr, T. A., Farmer, S. J., Glazer, S., Gudanowski, D. M. y Nair, V. N. (2003). The enigma of social support and occupational stress: Source congruence and gender role effects. Journal of Occupational Health Psychology, 8, 220-231.

Blanch, A. y Aluja, A. (2009). Validation study of the Spanish version of the Work-Family Conflict Questionnaire (CCTF). Spanish Journal of Psychology, 12(2), 746-755.

Boyar, S. L., Maertz, C. P. y Pearson, A. W. (2005). The effects of work-family conflict and familywork conflict or non-attendance behaviours. Journal of Business Research, 58(7), 919-925.

Burke, R. J. y Greenglass, E. (2001). Work stress, role conflict, social support and psychological burnout among teachers. Psychological Reports, 73, 371-380.

Cantera, L. M., Cubells, E., Martínez, L., Blanch, M. y Josep M. (2009). Work, Family, and Gender: Elements for a Theory of Work-Family Balance. The Spanish Journal of Psychology, 12(2), 641-647.

Carlson, D. S., Kacmar, K. M. y Williams, L. J. (2000). Construction and Initial Validation of a Multidimenasional Measure of Work-Family Conflict. Journal of Vocational Behavior, 56(2), 249-276.

Cohen, J., Cohen, P., West, S. G. y Aiken, L. S. (2003). Applied multiple regression/correlation analysis for the behavioral sciences ( $3^{\mathrm{a}}$ edición). Mahwah, N. J.: Lawrence Erlbaum Associates.

Encuesta de Calidad de Vida Europea (2012). Recuperado de: http://www.eurofound.europa.eu/ pubdocs/2012/64/en/1 EF1264EN.pdf.

Gil-Monte, P. R. (2002). Validez Factorial de la adaptación al español del Maslach Burnout Inventory-General Survey. Salud Pública de México, 44(1), 33-40.

Greenhaus, J. H. y Beutell, N. (1985). Sources of conflict between work and family roles. Academy of Management Review, 10(1), 76-88.

Haar, J. M. (2004). Work-family conflict and turnover intention: Exploring moderation effect of perceived work-family support. New Zealand Journal of Psychology, 33(1), 35-39.

Halbesleben, J. R. B. (2006). Sources of Social Support and Burnout: A Meta-Analytic Test of the Conservation of Resources Model. Journal Applied Psychology, 91(5), 1134-1145.

Hobfoll, S. E. (1989). Conservation of Resources Theory: A New Attempt at Conceptualizing Stress. American Psychologist, 44(3), 513-524.

Kahn, R. L. y Byosiere, P. (1992). Stress in organizations. En M.D. Dunnette y L.M. Hough (eds.), Handbook of industrial and organizational psychology (2a ed., Vol. 3, pp. 571-650). Palo Alto, California: Consulting Psychologists Press.

King, L. A., Mattimore, L. K., King, D. W. y Adams, G. A. (1995). Family support inventory for workers: a new measure or perceived social support from family members. Journal of Organizational Behaviour, 16(3), 235-258.

Korabik, K., McElwain, A., y Chappel, D. B. (2008). Integrating Gender-related issues into Research on Work and Family. En K. Korabik, D. Lero y D. Whitehead (Eds). Handbook of Work-Family Integration: Research, Theory and Best Practices. (pp. 215-232). San Diego, California: Elsevier.

Kossek, E. E. y Ozeki, C. (1999). Bridging the work-family policy and productivity gap: a literature review. Community, Work \& Family, 2 (1), 7-30. 
Lapierre, L. M. y Allen, T. D. (2006). Work-supportive family, family-supportive supervision, use of organizational benefits, and problem-focused coping: Implications for work-family conflict and employee well-being. Journal of Occupational Health Psychology, 11 (2), 169-181.

Major, D. A. y Germano, L. M. (2006). The changing nature of work and its impact on the workhome interface. En F. Jones, R. Burke, y M. Westman (eds), Work-Life Balance: A psychological Perspective (pp. 13-38). Londres: Psychology Press.

Maslach, C., Jackson, S. E. y Leiter, M. P. (1996). Maslach Burnout Inventory manual (3 ${ }^{\mathrm{a}}$ edición). Palo Alto, California: Consulting Psychologists Press.

Matthews, R. A., Bulger, C. A. y Barnes-Farrell, J. L. (2010). Work social supports, role stressors, and work-family conflict: The moderating effect of age. Journal of Vocational Behavior, 76(1), 78-90.

McElwain, A. K., Korabik, K. y Rosin, H. M. (2005). An examination of Gender differences in Work-Family Conflict. Canadian Journal of Behavioural Science, 37(4), 283-298.

Mesmer-Magnus, J. R. y Viswesvaran, C. (2005). Convergence between measures of work-to-family and family-to-work conflict: A meta-analytic examination. Journal of Vocatonal Behavior, 67(2), 215-232.

Osca, A. y Martínez-Pérez, M. D. (2002). Estudio Psicométrico del «Inventario de Apoyo Familiar para Trabajadores de King, Mattimore, King y Adams (1995). Psicotema, 14(2), 310-316.

Palladino Schultheiss, D. E. (2006). The interface of work and family life. Professional Psychology: Research and Practice, 37(4), 334-341.

Parasuraman, S., Greenhaus, J. H. y Granrose, C. S. (1992). Role stressors, social support, and well-being among two-career couples. Journal of Organizational Behavior, 13(4), 339-356.

Pleck, J. H. (1977). The work-family role system. Social Problems, 24, 417-427.

Powell, G.N., y Greenhaus, J.H. (2010). Sex, Gender, and Decisions at the Family-Work Interface. Journal of Management, 36(4), 1011-1039.

Purvanova, R. K. y Muros, J. P. (2010). Gender differences in burnout: A meta-analysis. Journal of Vocational Behavior, 77(2), 168-185.

Renes, V. (2009) (coord.). VI Informe Foessa sobre la Exclusión y el Desarrollo social. Madrid: Fundación Foessa Cáritas.

Rupert, P. A., Stevanovic, P. y Hunley, H. A. (2009). Work-family conflict and burnout among professional psychologists. Professional Psychology: Research \& Practice, 40(1), 54-61.

Schaufeli, W. B. y Bakker, A. B. (2004). Job demands, job resources, and their relationship with burnout and engagement: a multi-sample study. Journal of Organisational Behavior, 25(3), 293-315.

SIUSS (2009). Departamento de Política Social, Familias e Infancia. Ministerio de Sanidad, Servicios Sociales e Igualdad. En: www.msssi.gob.es/en/politicaSocial/inclusionSocial/

Tang, H., Ma, H., Wang, M. y Wang, B. (2009). A study of the relationship between Chinese clinicians? work-family conflict and job burnout. 2009 ISECS International Colloquium on Computing Communication Control and Management.

Viswesvaran, C., Sánchez, J. y Fisher, J. (1999). The role of social support in the process of work stress: a meta-analysis. Journal of Vocational Behavior, 54(2), 314-34.

Voydanoff, P. (2008). A Conceptual Model of the Work-Family Interface. En K. Korabik, D.S. Lero y D.L. Whitehead (Eds) (2008). The handbook of work-family integration: Theories, research \& best practices (pp. 37-56). San Diego, California: Elsevier.

Westman, M. y Etzioni, D. (2002). The Impact of Short Overseas Business Trips on Job Stress and Burnout. Applied Psychology, 52(4), 582-592.

Witt, L. A. y Carlson, D. S. (2006). The work-family interface and job performance: Moderating effect of conscientiousness and perceived organizational support. Journal of Occupational Health Psychology, 11(4), 342-357.

Xie, Y., Zeng, C. y Shi, K. (2007). The Effects of Work - Family Conflict on Telecom Employees' Job Burnout and General Health. Psychological Science, 30(4), 940-943

Zedeck, S. (1971). Problems with the use of «moderator» variables. Psychological Bulletin, 76, 295-310. 